\title{
Hyponatremia and Cerebral Edema Associated with Radioiodine Therapy for Papillary Thyroid Carcinoma in a Filipino
}

\author{
Monica Therese C. Cabral ${ }^{1}$, Brian Michael I. Cabral ${ }^{2}$, Iris Thiele C. Isip-Tan ${ }^{1}$ \\ ${ }^{1}$ Section of Endocrinology, Diabetes and Metabolism, Department of Medicine, University of the Philippines - Philippine General Hospital \\ ${ }^{2}$ Section of Nephrology, Department of Medicine, University of the Philippines - Philippine General Hospital
}

\begin{abstract}
Among the serious complications associated with radioiodine therapy (RAI) for thyroid cancer, cerebral edema is uncommon and has been reported previously in cases of swelling of brain metastases. This case is of a patient with papillary thyroid carcinoma who complained of nausea and vomiting after RAI and was then found unconscious the next day. Laboratory results showed electrolyte imbalances including hyponatremia and cranial imaging only revealed cerebral edema, and she regained consciousness after sodium correction. The etiology of the cerebral edema here is likely multifactorial - due to hyponatremia from hypothyroidism, aggravated by vomiting, copious water intake and a low sodium diet.
\end{abstract}

Keywords: hyponatremia, hypothyroidism, radioiodine

\section{Background}

In the treatment of well-differentiated thyroid cancer, radioiodine (I-131) therapy (RAI) for thyroid remnant ablation is safe and complications are usually mild and tolerable. More serious complications after RAI are rare but they can cause significant morbidity and mortality. The most critical acute complications are cerebral edema or hemorrhage due to swelling of brain metastases. However in this case, alteration of sensorium and cerebral edema occurred in the absence of brain lesions, and other factors may have been contributory. This is important to consider when faced with a patient who loses consciousness after RAI.

\section{Case report}

A 30-year-old female presented with a slowly growing anterior neck mass over the course of 2 years. She was diagnosed with nodular non-toxic goiter and subsequently had a subtotal thyroidectomy. Final histopathologic examination however revealed papillary thyroid carcinoma and a completion thyroidectomy with selective node dissection was then performed (Stage I, T3N0M0, <45 years old). After a few months the patient was admitted for RAI after cessation of levothyroxine (LT4) for 3 weeks and being advised to limit iodine intake in accordance with local guidelines. ${ }^{1}$ On the day of admission she complained of some light-headedness, which she

\author{
ISSN 0857-1074 \\ Printed in the Philippines \\ Copyright (C) 2012 by the JAFES \\ Received March 30,2012. Accepted: April 11, 2012.
}

This case report has been presented as a poster entitled "Cerebral edema: An unusual complication after radioiodine therapy for papillary thyroid carcinoma" at the Philippine Society of Endocrinology and Metabolism Annual Convention in Manila, Philippines, March 2011; Endocrine Society 93 ${ }^{\text {rd }}$ Annual Meeting in Boston, Massachusetts, June 2011; and at the 16 $6^{\text {th }}$ Congress of the ASEAN Federation of Endocrine Societies, Ho Chi Minh City, Vietnam, November 2011. attributed to ongoing menstruation. Pregnancy test was negative while vital signs were stable and physical examination unremarkable and ${ }^{131}$ I $3.7 \mathrm{GBq}(100 \mathrm{mCi})$ was administered. Six hours later, she complained of nausea and had 2 episodes of vomiting. On the second hospital day, nausea and vomiting persisted, although some relief was obtained with anti-emetics. She reported tolerance of small feedings and compliance with liberal fluid intake. She was normotensive and did not appear to be dehydrated. Two hours after last being seen however, she was found unconscious and could only be minimally roused with noxious stimuli. Vital signs were within normal limits and physical examination was unrevealing, with no evidence of trauma. Neurologic examination did not reveal any lateralizing signs. Pupils were equal and briskly reactive, reflexes were symmetric and extensor toe sign was negative. There was no note of abnormal posturing, involuntary movements or withdrawal to painful stimuli. Dose rate at a distance of 1 meter from the patient was $10 \mathrm{mR} / \mathrm{hr}$.

\section{Differential diagnosis}

Differential diagnoses for her altered sensorium at this time included hypotension due to dehydration from vomiting and possible head trauma. Metabolic causes could have been either from hypoglycemia due to poor intake and excessive vomiting or hyponatremia secondary

Corresponding author: Monica Therese Cating-Cabral, MD

Address: Philippine General Hospital

Taft Avenue, Manila 1000

Telephone number: +632-554-8400 loc 3230 or +63917-622-2725

E-mail address: mitos@hotmail.com 
to the hypothyroidism induced by withholding LT4. The most serious concern was possible cerebral edema due to swelling of undetected brain metastases.

\section{Investigations}

Initial capillary blood glucose was $4.38 \mathrm{mmol} / \mathrm{L}$ and after administration of 1 vial of intravenous $50 \%$ dextrose, her blood glucose was rechecked and noted to be 12.21 $\mathrm{mmol} / \mathrm{L}$. The patient did not regain consciousness. Multiple electrolyte abnormalities were found, including hyponatremia (sodium:118 mmol/L, NV:136-145 mmol/L), hypokalemia (potassium:2.5 mmol/L, NV:3.5-5.1 mmol/L), hypomagnesemia (magnesium:0.57 mmol/L, NV:0.7-1.0 $\mathrm{mmol} / \mathrm{L}$ ) and hypocalcemia (calcium:1.71 $\mathrm{mmol} / \mathrm{L}$, $\mathrm{NV}: 2.12-2.52 \mathrm{mmol} / \mathrm{L}$ ). Serum osmolality was low at 251 $\mathrm{mmol} / \mathrm{kg}$. Urine specimens for electrolytes and osmolality were not obtained due to the patient's elevated radiation levels. A 12-lead electrocardiogram showed occasional premature ventricular contractions (PVCs) with no other specific changes.

While obtaining a computed tomographic (CT) scan, a lead gown and thyroid shield were placed over the patient. This was in accordance with radiation safety practices, providing healthcare providers with adequate shielding from radiation as the patient had just received RAI the day before. Plain cranial CT scan showed hypodensities in the frontal subcortical regions that were interpreted as acute bilateral frontal infarcts. (Figure 1-A). Mild pontine and cerebral edema were also noted. (Figures 2-A and 2-B).

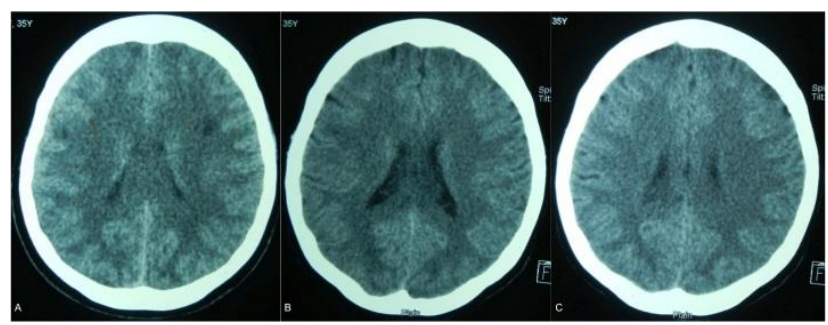

Figure 1. Plain cranial CT scan showing acute bilateral frontal infarcts $(A)$ and subsequent resolution (B and $C)$.

\section{Treatment}

Given the possible etiologies, the most critical was that of cerebral edema due to brain metastases and intravenous (IV) dexamethasone was started as soon as the patient was found unconscious. The Neurology service was also consulted, and once the CT scan was obtained, furosemide and mannitol were given for the cerebral edema. Intravenous correction was started for the electrolyte deficiencies.

\section{Outcome and follow-up}

The patient regained consciousness the next day, 16 hours after the being found unresponsive. She could not recall the previous events, except for the sensation of dizziness and episodes of vomiting. She admitted to drinking numerous glasses of water but could not recall the exact amount. The patient was found to have 2 almost-empty 5-gallon containers of drinking water in her hospital room which were full on admission, with no spillage was observed. Physical and neurologic examination did not reveal any lateralizing signs suggestive of an acute cerebrovascular event. Serum sodium was corrected to $128 \mathrm{mmol} / \mathrm{L}$ and subsequently to $130 \mathrm{mmol} / \mathrm{L}$ by the fourth hospital day, with the other electrolytes corrected to within normal range. A follow-up plain cranial CT scan showed no evidence of the previously seen frontal infarcts (Figures 1-B and 1-C) with resolution of the pontine and cerebral edema (Figures 2-C and 2-D). A cranial CT scan with contrast was also done and this did not reveal any other abnormalities. A post-ablative whole body scan done 7 days after RAI showed only functioning residual thyroid tissue in the anterior neck and no evidence of metastases to the brain (Figure 3).

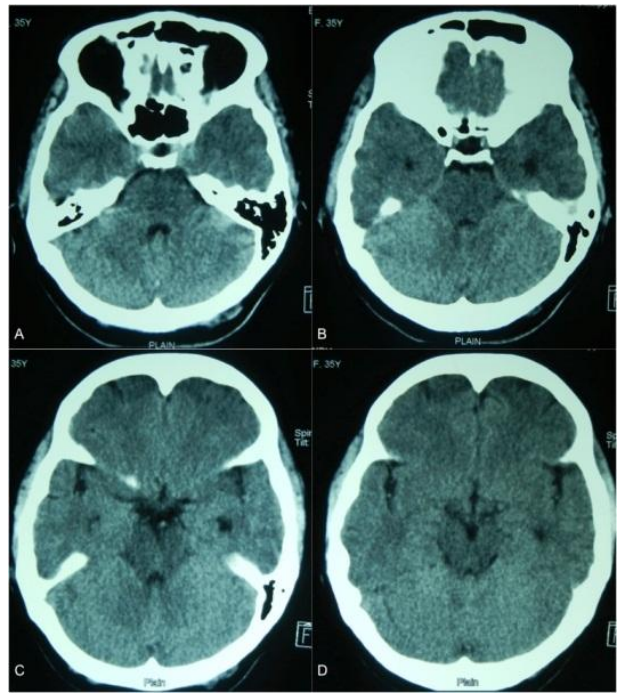

Figure 2. Plain cranial CT scan showing mild pontine and cerebral edema (A and $B$ ) and resolution after electrolyte correction ( $C$ and $D)$.

Based on these findings and the patient's response to sodium correction, it was concluded that the cerebral edema was secondary to hyponatremia probably due to hypothyroidism and exacerbated by a low iodine and sodium diet, gastrointestinal losses due to acute radiation sickness and excessive fluid intake.

\section{Discussion}

Serious complications associated with RAI are unusual, but if they do occur it is after large single administrations of RAI at doses above ${ }^{131}$ I 7.4 GBq (200 mCi) or when large cumulative amounts of RAI have been given. ${ }^{2,3}$ In this case, this was the patient's first, relatively low dose of RAI. 


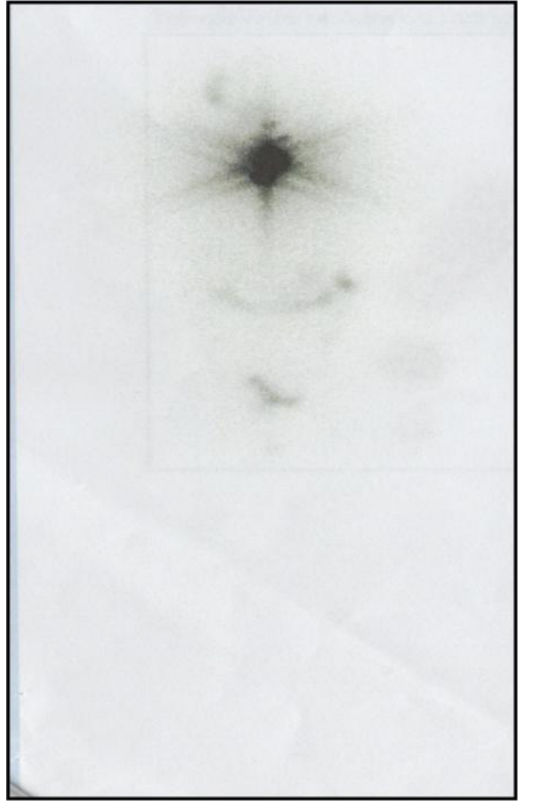

Figure 3. Post-therapy whole body scan showing functioning residual thyroid tissue in the anterior neck

\section{Early complications}

Gastrointestinal symptoms be due to acute radiation sickness may occur as early as 2-12 hours and up to 36 hours after RAI and typically lasts 1-2 days. Symptoms include fatigue, headache, nausea and vomiting which are controlled with anti-emetics. The reported incidence varies from $36-73 \%$, but vomiting occurs in no more than $5 \% .3,4$

\section{Central nervous system complications}

Radiation-induced swelling may also affect metastatic tissue. Aside from bony and soft tissue, this may occur in the spinal cord or brain, causing pain, edema, hemorrhage and compressive symptoms. . $^{2,6,7}$ The brain accounts for only $1 \%$ of the $10 \%$ of distant metastases that occur in patients with well-differentiated thyroid carcinoma, ${ }^{7-9}$ but the effects of radiation-induced swelling can be devastating. There are a few reports on serious central nervous system complications after RAI, including a case of hemorrhage of brain metastases and 2 of cerebral edema, with 1 being fatal.3,5,6 It has been recommended pre-treatment prior to RAI with corticosteroids and mannitol may reduce the risk for this complication. .,10,11 $^{2}$

\section{Hyponatremia and hypothyroidism}

Symptomatic hyponatremia has been reported to complicate RAI, although usually in the period prior to treatment during withdrawal of LT4. Reported cases complained of dizziness and confusion just before RAI, and all were found to have low sodium levels which were either treated with saline, fluid restriction or liothyronine (LT3) prior to RAI. ${ }^{12,13}$ In another reported case, the symptoms of hyponatremia manifested almost 2 weeks after RAI with LT4 replacement already resumed. ${ }^{14}$ In all of these cases the patients were over 60 years old and had been on LT4 withdrawal and low iodine diets in preparation for RAI.

For patients undergoing RAI, current guidelines recommend LT4 withdrawal for at least 2-3 weeks or LT3 treatment for 2-4 weeks and LT3 withdrawal for 2 weeks to achieve hypothyroidism with a TSH level of $>30$ mIU/L. ${ }^{11}$ Hypothyroidism has been noted to induce hyponatremia, although the mechanism by which this occurs is incompletely understood. Hyponatremia itself is the most commonly encountered electrolyte disorder in hospitalized patients, and is more of a disorder in water rather than sodium balance. ${ }^{15}$ In hypothyroidism, the principal abnormality in patients with normal fluid intake appears to be an inability to maximally suppress antidiuretic hormone (ADH). ${ }^{16}$ There is also a decrease in renal blood flow and glomerular filtration and this can directly diminish free water excretion by diminishing water delivery to the diluting segments. ${ }^{17,18}$ These however do not lead to significant hyponatremia unless there are other contributory factors such as diuretic use, excessive extra-renal losses as in diarrhea and vomiting or large volumes of fluid intake, ${ }^{18}$ as in our case. Reduced cardiac output in hypothyroidism may also play a role, stimulating volume-mediated ADH release via the carotid sinus baroreceptors. ${ }^{17,19}$

It is unclear if there truly is a relationship between hypothyroidism and hyponatremia. Studies in hypothyroid children and adults have shown that low serum sodium concentrations were not altered despite LT4 correction. $^{20-22}$ In one report of almost 1,000 hypothyroid patients, none were found to have serum sodium concentrations $<120 \mathrm{mmol} / \mathrm{L}$ although this was present in 2 controls. ${ }^{23}$ Given this data it would appear that the presence of a low serum sodium in a hypothyroid patient would require further investigation into other possible causes for hyponatremia.

\section{Hyponatremia and low iodine diets}

Iodine depletion has been found to allow for increased delivery of the radiation dose in thyroid cancer ablative therapy. ${ }^{24}$ This is achieved by a low iodine diet $(<50 \mathrm{mg} / \mathrm{d}$ of dietary iodine) for 1-2 weeks prior to RAI, especially in patients with high iodine intake. Patient should also avoid any iodine contamination (e.g., intravenous contrast, amiodarone use) to increase the effective radiation dose. ${ }^{11}$

Unfortunately, many patients equate a low iodine diet with a low salt diet. ${ }^{13}$ In our setting where it is mandated by law that all salt be iodized, ${ }^{25}$ it is difficult to obtain noniodized salt and this leads to the avoidance of salt altogether. In states of low solute intake, the ability to generate and therefore excrete free water becomes limited if solute excretion is markedly decreased, which can further worsen hyponatremia, if present. ${ }^{26}$ In susceptible patients with hypothyroidism, such as the elderly, serum 
sodium levels should be measured in the event that they exhibit symptoms of hyponatremia. ${ }^{13}$

\section{Hyponatremia and polydipsia}

It is possible that excessive fluid intake may have contributed to the condition of the patient in this case. Patients are advised to drink water liberally and urinate frequently to avoid unnecessary radiation to the bladder and pelvic area after RAI therapy.,27 The patient admitted to drinking large amounts, but the actual volume of water intake was not documented. Hyponatremia related to polydipsia is generally considered to be an acute dilutional state in which water intake overwhelms renal excretory capacity. It is also said to be a mixed disorder of increased intake of water and loss of solute. ${ }^{28}$ In normal subjects, 10 to 15 liters of urine may be excreted per day, a response that is mediated by suppression of $\mathrm{ADH}$ secretion and the subsequent formation of a dilute urine. Thus, if ADH regulation were intact, intake of about 10 to 12 liters of water should not lead to clinically important disturbances in the plasma sodium concentration. ${ }^{29}$ Water intoxication and hyponatremia could occur however if intake were massively increased, ${ }^{28}$ or if there was volume depletion, use of diuretics, or the presence of co-existing disturbances in renal, endocrine, cardiac and hepatic function. In these situations, amounts of fluid that are below the water excretory capacity of the kidneys can already cause hyponatremia. ${ }^{25}$

\section{Conclusion}

Loss of consciousness and the development of cerebral edema after RAI is uncommon and its occurrence should alert physicians to the possibility of swelling of undetected brain metastases. However in hypothyroid patients, this may also occur in the setting of hyponatremia, and in this case, was exacerbated by vomiting, a low sodium diet and excessive fluid intake.

This was a young patient who presented with symptomatic hyponatremia, which is unusual in her age group, as this has only been observed in elderly patients placed on low iodine diets who may equate this with a low sodium diet. Proper instruction on dietary restriction should be discussed with patients prior to RAI, and monitoring of serum sodium levels should be considered for symptomatic or elderly patients. Once RAI has been administered, patients are also advised liberal fluid intake during their hospital stay but they should avoid excessive amounts.

\section{Acknowledgements}

Thank you to Dr. Ruben Ogbac, Dr. Lorraine Almelor and the nurses at the Cancer Institute of the Philippine General Hospital for their assistance in the management of this case.

\section{References}

1. PGH Working Group on Thyroid Cancer. Clinical Practice Guidelines of the Philippine General Hospital for the management of thyroid nodules and well-differentiated thyroid carcinoma. Acta Medica Philippina. 2008;42(1):56-67.

2. Mazzaferri EL, Kloos RT. Current approaches to primary therapy for papillary and follicular thyroid cancer. J Clin Endocrinol Metab. 2001;86:1447-63.

3. Hurley JR, Becker DV. Nuclear Medicine Annual. New York:Raven Press 1983.

4. Van Nostrand D, Neutze J,Atkins F. Side effects of "rational dose" iodine131 therapy for metastatic well-differentiated thyroid carcinoma. J Nucl Med 1986;27(10):1519-27.

5. Holmquest DL, Lake P. Sudden hemorrhage in metastatic thyroid carcinoma of the brain during treatment with iodine-131. J Nucl Med. 1975;17(4):307-9.

6. Datz FL. Cerebral edema following iodine-131 therapy for thyroid carcinoma metastatic to brain. J Nucl Med. 1986;27(5):637-40.

7. Chiu AC, Delpassand ES, Sherman SI. Prognosis and treatment of brain metastases in thyroid carcinoma. J Clin Endocrinol Metab. 1997;9(11):363742.

8. Schlumberger, MJ. Papillary and follicular thyroid carcinoma. N Engl J Med. 1998;338(5):297-306.

9. Durante C, Haddy N, Baudin E, et al. Long-term outcome of 444 patients with distant metastases from papillary and follicular thyroid carcinoma: Benefits and limits of radioiodine therapy. J Clin Endocrinol Metab. 2006;91(8):2892-9.

10. Maxon HR III, Smith HS. Radioiodine-131 in the diagnosis and treatment of metastatic well differentiated thyroid cancer. Endocrinol Metab Clin North Am. 1990;19:685-718.

11. American Thyroid Association (ATA) Guidelines Taskforce on Thyroid Nodules and Differentiated Thyroid Cancer. Revised American Thyroid Association management guidelines for patients with thyroid nodules and differentiated thyroid cancer. Thyroid. 2009;19(11):1167-214.

12. Krishnamurthy VR, McDougall IR. Severe hyponatremia: A danger of lowiodine diet. Thyroid. 2007;17(9):889-92.

13. Shakir MK, Krook LS, Schraml FV, Hays JH, Clyde PW. Symptomatic hyponatremia in association with a low-iodine diet and levothyroxine withdrawal prior to I131 in patients with metastatic thyroid carcinoma. Thyroid. 2008;18(7):787-92.

14. Nozu T, Yoshida Y, Ohira M, Okumura T. Severe hyponatremia in association with $\mathrm{I}(131)$ therapy in a patient with metastatic thyroid cancer. Intern Med. 2011;50(19):2169-74.

15. Hoorn EJ, Lindemans J, Zietse R. Development of severe hyponatremia in hospitalized patients: Treatment-related risk factors and inadequate management. Nephrol Dial Transplant. 2006;21(1):70-6.

16. Skowsky MR, Kikuchi TA. The role of vasopressin in the impaired water excretion of myxedema. Am J Med. 1978;64(4):613-21.

17. Hoorn EJ, Zietse R. Hyponatremia revisited: Translating physiology to practice. Nephron Physiol. 2008;108(3):46-59.

18. Schrier RW. Body water homeostasis: Clinical disorders of urinary dilution and concentration. J Am Soc Nephrol. 2006;17(7):1820.

19. Hanna FW, Scanlon MF. Hyponatremia, hypothyroidism, and role of arginine-vasopressin. Lancet. 1997;350(9080):755.

20. Kilpatrick, ES. Disorders of sodium balance: Hypothyroidism and hyponatremia: an old wives' tale? BMJ. 2006;332(7545):854.

21. Croal BL, Blake AM, Johnston J, Glen AC, O'Reilly DS. Absence of relation between hyponatremia and hypothyroidism. Lancet. 1997;350(9088):1402.

22. Baajafer FS, Hammami MM, Mohamed GED. Prevalence and severity of hyponatremia and hypercreatininemia in short-term uncomplicated hypothyroidism. J Endocrinol Invest. 1999;22(1):35-9.

23. Warner MH, Holding S, Kilpatrick ES. The effect of newly diagnosed hypothyroidism on serum sodium concentrations: A retrospective study. Clin Endocrinol (Oxf). 2006;64(5):598-9.

24. Maxon HR, Thomas SR, Boehringer A, et al. Low iodine diet in I-131 ablation of thyroid remnants. Clin Nucl Med. 1983;8(3):123-6.

25. Philippine Senate. Republic Act 8172 - An act promoting salt iodization nationwide and for related purposes. Manila:The Senate;1995.

26. Thaler SM, Teitelbaum I, Berl T. "Beer potomania" in non-beer drinkers: Effect of low dietary solute intake. Am J of Kidney Dis. 1998;31(6):1028-31.

27. The American Thyroid Association Taskforce on Radioiodine Safety. Radiation safety in the treatment of patients with thyroid diseases by radioiodine 131-I: Practice recommendations of the American Thyroid Association. Thyroid. 2011;21(4):335.

28. Musch $\mathrm{W}$, Xhaet $\mathrm{O}$, Decaux $\mathrm{G}$. Solute loss plays a major role in polydipsiarelated hyponatremia of both water drinkers and beer drinkers. QJM. 2003;96(6):421-6.

29. Gillum DM, Linas SL. Water intoxication in a psychotic patient with normal renal water excretion. Am J Med. 1984;77(4):773-4. 